\title{
Laser-assisted (e, 2e) reaction in one-electron atoms and ions
}

\author{
S-M Li ${ }^{1,2}, \mathbf{J}$ Berakdar ${ }^{2}$, S-T Zhang ${ }^{1,3}$ and $\mathbf{J}$ Chen $^{3,4}$ \\ ${ }^{1}$ Open Laboratory of Bond Selective Chemistry, Department of Modern Physics, University of \\ Science and Technology of China, PO Box 4, Hefei, Anhui 230026, People's Republic of China \\ ${ }^{2}$ Max-Planck Institut für Mikrostrukturphysik, Weinberg 2, 06120 Halle, Germany \\ ${ }^{3}$ China Center of Advanced Science and Technology (World Laboratory), PO Box 8730, \\ Beijing 100080, People's Republic of China \\ ${ }^{4}$ Department of Modern Physics, University of Science and Technology of China, PO Box 4, \\ Hefei, Anhui 230026, People's Republic of China \\ E-mail: jber@mpi-halle.de
}

Received 27 December 2004

Published 8 April 2005

Online at stacks.iop.org/JPhysB/38/1291

\begin{abstract}
We study the ionization of a hydrogenic target by the impact of moderate energy electrons and in the presence of a laser field. This process, generally referred to as the laser-assisted (e, 2e), is treated non-relativistically as follows: we evaluate the $S$-matrix elements using Volkov and Coulomb-Volkov wavefunctions for describing respectively the continuum states of the scattered and the ejected electrons. The dressed state of the target is described by a time-dependent wavefunction derived from a perturbation treatment (of the laser field). With the aid of numerical results we explore the dependences of the fully differential cross sections on the laser field properties such as the strength, the frequency and the polarization direction, as well as on the continuum electron energies and their emission angles. Furthermore, we assess the interplay between the laser influence and the role of scattering from the residual ion by comparing the cross sections from (one-electron) ionic targets and varying the charge state of the residual ion.
\end{abstract}

(Some figures in this article are in colour only in the electronic version)

\section{Introduction}

The treatment of electronic collision processes in external fields, and in particular in laser fields is fundamentally important and is also of interest for some applications. From a fundamental point of view, the investigation of the few-particle dynamics in a laser background serves as a paradigm example for studying the time-dependent behaviour of correlated few-body systems far from equilibrium. On the other hand, a detailed knowledge of various aspects of 
laser-assisted electronic collisions is of considerable importance for a number of applications such as plasma heating and for semiconductor physics where the electron-electron collision rate (and hence the transport properties of the system) can be steered by applying an external laser field. A general overview on the field of laser-assisted atomic collisions is provided by the book of Mittleman [1] and some recent reviews [2-4], whereas the laser-driven electron and hole dynamics in semiconductors is treated in the book by Haug and Jauho [5]. With the recent development in laser technology combined with the impressive advances in multi-particle detection techniques it is conceivable that the experimental realization of the laser-assisted electron-atom ionizing collision will be accomplished in the near future [6, 7]. Hence it is timely and desirable to study theoretically the various facets of the energy and angular resolved cross section for the electron-impact ionization of hydrogenic targets in the presence of a laser background. In the literature this process is called laser-assisted (e, 2e) and is in the focus of the present work. The (e, 2e) process for the laser-free case is well-studied by now (cf e.g. [8] and references therein). An addition of a laser field leads to essential modifications (dressing) of the target states and it also influences decisively the state of the continuum electrons, as detailed below. Previously, several publications have dealt with the (e, 2e) reaction in a laser field: at an early stage of this research field the laser modulation of the electron bound state was neglected, and the target state was described simply by the field-free wavefunction [9]. Later on Joachain and coworkers investigated the case of an atomic hydrogen [10, 11] and of helium $[12,13]$. In these studies processes have been considered where few photons are involved and the $S$-matrix elements were evaluated in the first Born approximation (FBA) for ionization. While there is no formal justification for employing the first Born approximation for processes involving (infinite range) Coulomb potentials it turns out that, for a particular choice of the scattering geometry, the FBA provides a useful tool for capturing the qualitative trends in the measured cross sections. The choice one usually makes is that the wave vectors of the incoming and outgoing electrons are in one plane and the projectile electron is fast (few times the ionization potential). Furthermore the momentum transfer is small. This particular scattering geometry is called the co-planar asymmetric geometry. In [10-13] the states of the projectile electron are then described by Volkov wavefunctions [14] whereas for the slow ejected electron the combined influence of the laser field and the Coulomb field of the residual ion is taken into account [10]. The influence of the laser on the target states is treated using a first-order perturbation approach. The measurable quantity which is calculated by theory is the so-called triplet differential cross section, i.e. the coincident differential cross section that depends on the energies and the emission solid angles of the continuum electrons. It has been found that when few multiphoton processes are involved, the qualitative behaviour of the cross sections as a function of the solid angles of the final state electrons and the electron energies are modified markedly by the presence of the laser field. The cross sections were also found to depend decisively on the dressing of the atomic target states. On the other hand for strong lasers, readily available nowadays, a large number of multiphoton processes may take place during such collisions. Hence, we present in this paper our FBA calculations for the cross section of the laser-assisted $(\mathrm{e}, 2 \mathrm{e})$ from hydrogenic targets including all multiphoton processes. In addition, our choice to study hydrogenic ions allows us to test for the relative influence of the Coulomb field of the target nucleus (by varying the value of the charge of the residual ion).

This paper is organized as follows: in section 2 we describe the method used to evaluate the scattering matrix. In section 3, we discuss the numerical results for the cross section versus the electrons' scattering angles and the electrons' energies. The influence of the laser parameters (e.g., the intensity, the frequency and the direction of the polarization vector) on the cross sections summed over multiphoton processes is discussed. Section 4 summarizes 
and concludes the present work. Unless specifically stated, atomic units (a.u.) $\hbar=m=e=1$ are used throughout.

\section{Theory}

During a laser-assisted electron-impact ionization process $l$ photons may be exchanged with the laser field. Schematically, the (e, 2e) process in a laser background can be written as

$$
\mathrm{e}^{-}\left(\mathbf{k}_{i}\right)+A^{+Z-1}(1 \mathrm{~s})+l \omega \longrightarrow A^{+Z}+\mathrm{e}^{-}\left(\mathbf{k}_{f}\right)+\mathrm{e}^{-}\left(\mathbf{k}_{e}\right) .
$$

Here $Z$ is the charge of the residual ion. The target $A^{+Z-1}(1 \mathrm{~s})$ is a one-electron ion residing initially (in the absence of the laser) in the $1 \mathrm{~s}$ ground state. The vectors $\mathbf{k}_{i, f}$ stand respectively for the initial and the final momentum of the projectile electron and $\mathbf{k}_{e}$ is the momentum vector of the slow ejected electron $\left(k_{f}>k_{e}<k_{i}\right)$. The laser field is such that it can be treated classically as a single mode and (over the extent of the target) spatially homogeneous, linearly polarized field. Within the dipole approximation we write for the electric field $\mathcal{E}(t)$ of the laser

$$
\mathcal{E}(t)=\mathcal{E}_{0} \sin \omega t
$$

where $\mathcal{E}_{0}$ is the electric field amplitude vector and $\omega$ is the frequency.

As mentioned in the introduction we will consider the asymmetric co-planar geometry and operate within the FBA. This means that the states of the fast projectile electron both in the initial $\left(\chi_{\mathbf{k}_{i}}\right)$ and in the final $\left(\chi_{\mathbf{k}_{f}}\right)$ states are described by Volkov wavefunctions [14, 15], i.e.,

$$
\begin{aligned}
& \chi_{\mathbf{k}_{i}}\left(\mathbf{r}_{0}, t\right)=(2 \pi)^{-3 / 2} \exp \left\{\mathrm{i}\left[\mathbf{k}_{i} \cdot \mathbf{r}_{0}-\mathbf{k}_{i} \cdot \boldsymbol{\alpha}_{0} \sin \omega t-E_{i} t-\frac{1}{2 c^{2}} \int_{-\infty}^{t} A^{2}\left(t^{\prime}\right) \mathrm{d} t^{\prime}\right]\right\}, \\
& \chi_{\mathbf{k}_{f}}\left(\mathbf{r}_{0}, t\right)=(2 \pi)^{-3 / 2} \exp \left\{\mathrm{i}\left[\mathbf{k}_{f} \cdot \mathbf{r}_{0}-\mathbf{k}_{f} \cdot \boldsymbol{\alpha}_{0} \sin \omega t-E_{f} t-\frac{1}{2 c^{2}} \int_{-\infty}^{t} A^{2}\left(t^{\prime}\right) \mathrm{d} t^{\prime}\right]\right\},
\end{aligned}
$$

where $\mathbf{A}(t)=(c / \omega) \mathcal{E}_{0} \cos \omega t$ is the vector potential of the laser field ( $c$ is the light velocity), and $\boldsymbol{\alpha}_{0}=\mathcal{E}_{0} / \omega^{2} . E_{i, f}=k_{i, f}^{2} / 2$ are the projectile electron asymptotic energies in the absence of the laser. The position of the projectile electron $\mathbf{r}_{0}$ is measured with respect to the residual ion (which is assumed to be infinitely heavy as compared to the electron mass). The slow ejected electron is subjected to the combined influence of the laser field and the residual ion with charge $Z$. This continuum state we describe by the Coulomb-Volkov state $[9,16]$ that satisfies incoming spherical wave boundary conditions, i.e.,

$$
\begin{aligned}
\chi_{e}\left(\mathbf{r}_{1}, t\right)=(2 \pi)^{-3 / 2} \mathrm{e}^{\pi \xi / 2} \Gamma(1+\mathrm{i} \xi)_{1} F_{1}\left(-\mathrm{i} \xi, 1,-\mathrm{i}\left(k_{e} r_{1}+\mathbf{k}_{e} \cdot \mathbf{r}_{1}\right)\right) \\
\quad \times \exp \left\{\mathrm{i}\left[\mathbf{k}_{e} \cdot \mathbf{r}_{1}-\mathbf{k}_{e} \cdot \boldsymbol{\alpha}_{0} \sin \omega t-E_{e} t-\frac{1}{2 c^{2}} \int_{-\infty}^{t} A^{2}\left(t^{\prime}\right) \mathrm{d} t^{\prime}\right]\right\} .
\end{aligned}
$$

Here $\mathbf{r}_{1}$ is the position vector of the ejected electron with respect to the target, $\mathbf{k}_{e}$ is the wave vector of the ejected electron and $\xi=Z / k_{e}$ is the Sommerfeld parameter. ${ }_{1} F_{1}(a, b, z)$ is the confluent hypergeometric function in the notation of [17]. As will be discussed below, the electric field strength of interest here is usually smaller than an atomic unit, $\mathcal{E}_{0}<e / a_{0}^{2} \approx 5 \times 10^{9} \mathrm{~V} \mathrm{~cm}^{-1}$; where $e / a_{0}^{2}$ is a measure for the Coulomb field (for $Z=1$ ) which is experienced by an electron in the ground state. Hence for the treatment of the initially bound electronic states of the target $\psi_{0}\left(\mathbf{r}_{1}\right)$ when the laser is switched on, we treat the laser field perturbatively and find from the solution of the time-dependent Schrödinger equation for the dressed atom that [18],

$\psi_{0}\left(\mathbf{r}_{1}, t\right)=\left(1-\frac{\mathrm{i}}{\omega} \mathcal{E}_{0} \cdot \mathbf{r}_{1} \cos \omega t\right) \phi_{0}\left(\mathbf{r}_{1}\right) \exp \left\{-\mathrm{i}\left[W_{0} t+\frac{1}{2 c^{2}} \int_{-\infty}^{t} \mathrm{~d} t^{\prime} A^{2}\left(t^{\prime}\right)\right]\right\}$. 
The ground-state energy of the target is $W_{0}=-Z^{2} / 2$ a.u. and $\phi_{0}\left(\mathbf{r}_{1}\right)$ is the ground state in the absence of the laser, i.e.,

$$
\phi_{0}\left(\mathbf{r}_{1}\right)=\frac{\lambda^{3 / 2}}{\sqrt{\pi}} \mathrm{e}^{-\lambda r_{1}}, \quad(\lambda=Z) .
$$

The $S$-matrix for the laser-assisted $(\mathrm{e}, 2 \mathrm{e})$ reaction is given by the expression

$$
\begin{aligned}
S & =-\mathrm{i} \int_{-\infty}^{\infty} \mathrm{d} t\left\langle\chi_{\mathbf{k}_{f}}\left(\mathbf{r}_{0}, t\right) \chi_{e}\left(\mathbf{r}_{1}, t\right)\left|-\frac{Z}{r_{0}}+\frac{1}{r_{10}}\right| \chi_{\mathbf{k}_{i}}\left(\mathbf{r}_{0}, t\right) \psi_{0}\left(\mathbf{r}_{1}, t\right)\right\rangle \\
& =-\mathrm{i} 2 \pi \sum_{l=-\infty}^{\infty} T_{l} \delta\left(E_{f}+E_{e}-E_{i}-W_{0}+l \omega\right),
\end{aligned}
$$

where $\mathbf{r}_{10}=\mathbf{r}_{1}-\mathbf{r}_{0}$ is the inter-electronic distance. The transition matrix element, resolved with respect to multiphoton exchange processes, has the form

$$
\begin{aligned}
T_{l}=-\frac{\lambda^{3 / 2}}{\sqrt{2} \pi^{3}} & J_{l}\left(\mathbf{D} \cdot \boldsymbol{\alpha}_{0}\right) \mathrm{e}^{\pi \xi / 2} \Gamma(1-\mathrm{i} \xi) \frac{1}{\mathbf{q}^{2}} \frac{\partial}{\partial \lambda}\left\{\left.Z\left(1+\frac{l \omega}{\mathbf{D} \cdot \mathcal{E}_{0}} \mathcal{E}_{0} \cdot \frac{\partial}{\partial \gamma}\right)\right|_{\gamma=\mathbf{0}}\right. \\
& \left.\times \frac{\left[\gamma^{2}+\left(\lambda-\mathrm{i} k_{e}\right)^{2}\right]^{-\mathrm{i} \xi}}{\left[\left(\gamma+\mathbf{k}_{e}\right)^{2}+\lambda^{2}\right]^{1-\mathrm{i} \xi}}-\left(1+\frac{l \omega}{\mathbf{D} \cdot \mathcal{E}_{0}} \mathcal{E}_{0} \cdot \frac{\partial}{\partial \mathbf{q}}\right) \frac{\left[q^{2}+\left(\lambda-\mathrm{i} k_{e}\right)^{2}\right]^{-\mathrm{i} \xi}}{\left[\left(\mathbf{q}+\mathbf{k}_{e}\right)^{2}+\lambda^{2}\right]^{1-\mathrm{i} \xi}}\right\},
\end{aligned}
$$

in which $\mathbf{D}=\mathbf{k}_{e}+\mathbf{k}_{f}-\mathbf{k}_{i}$ measures the recoil ion momentum and $\mathbf{q}=\mathbf{k}_{f}-\mathbf{k}_{i}$ is the momentum transfer vector. In obtaining equations (8) and (9), we have used the following formulae for the Bessel functions [19]

$$
\mathrm{e}^{\mathrm{i} y \sin u}=\sum_{l=-\infty}^{\infty} J_{l}(y) \mathrm{e}^{\mathrm{i} l u}
$$

and

$$
J_{l-1}(y)+J_{l+1}(y)=\frac{2 l}{y} J_{l}(y),
$$

and the integration formula [20,21],

$$
\int \frac{\mathrm{e}^{-\mathrm{i}(\mathbf{k}+\mathbf{p}) \cdot \mathbf{r}-\lambda r}}{r}{ }_{1} F_{1}(\mathrm{i} \xi, 1, \mathrm{i}(k r+\mathbf{k} \cdot \mathbf{r})) \mathrm{d}^{3} \mathbf{r}=4 \pi \frac{\left[p^{2}+(\lambda-\mathrm{i} k)^{2}\right]^{-\mathrm{i} \xi}}{\left[(\mathbf{k}+\mathbf{p})^{2}+\lambda^{2}\right]^{1-\mathrm{i} \xi}} .
$$

As mentioned above, the measurable quantity that characterizes reaction (1) is the triple differential cross section (TDCS) which depends on the two solid electron emission angles and on the energy of one of the electrons. The TDCS for the net exchange of $l$ photons between the colliding system and the laser field is

$$
\frac{\mathrm{d}^{2} \sigma_{l}}{\mathrm{~d} \Omega_{f} \mathrm{~d} \Omega_{e} \mathrm{~d} E_{e}}=(2 \pi)^{4} \frac{k_{f} k_{e}}{k_{i}}\left|T_{l}\right|^{2} .
$$

The theory is applicable to situations where the velocities of the scattered and the ejected electrons are markedly different so that exchange effects between the two electrons are negligible. As demonstrated in the case of $(e, 2 e)$ in the absence of a laser field [22], the FBA is capable of reproducing the main features in the TDCS at moderate and high energies. These are the so-called binary and recoil peaks [23]. For a precise quantitative description one has however either to deal with higher order terms in the Born series or to abandon the use of a perturbative treatment in favour of more accurate, however computationally more expensive procedures. While the implementation of such procedures is performed successfully in the absence of the laser we are not aware that the laser-assisted (e, 2e) process has been tackled numerically in a non-perturbative manner. 


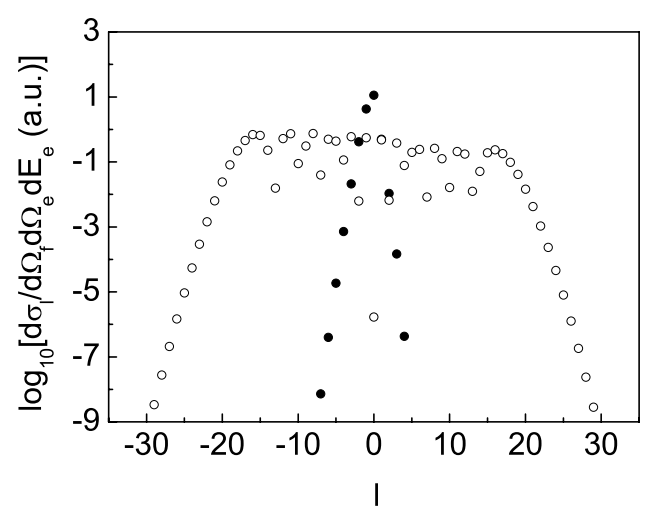

Figure 1. Multiphoton TDCS for the laser-assisted (e, 2e) from atomic hydrogen for the coplanar asymmetric geometry. The scattering angle of the fast emitted electron is $\theta_{f}=4^{\circ}$, the slow electron is ejected at the polar angle $\theta_{e}=45^{\circ}$. The azimuth angle is $\phi=0^{\circ}$. The kinetic energies of the incident and ejected electrons are respectively $E_{i}=150 \mathrm{eV}$ and $E_{e}=3 \mathrm{eV}$, the laser amplitude is $\mathcal{E}_{0}=5.0 \times 10^{7} \mathrm{~V} \mathrm{~cm}^{-1}$, and the frequency $\hbar \omega=1.17 \mathrm{eV}$ (Nd:YAG laser). Solid circles: the TDCS when the laser polarization vector is parallel to the incident electron momentum vector $\mathcal{E}_{0} \| \mathbf{k}_{i}$; hollow circles: the results when the laser polarization is perpendicular to $\mathbf{k}_{i}$, i.e. $\mathcal{E}_{0} \perp \mathbf{k}_{i}$.

\section{Results and discussion}

In this section, we present and analyse our findings for the TDCS of the laser-assisted $(\mathrm{e}, 2 \mathrm{e})$ reaction in the coplanar asymmetric geometry. Without loss of generality we assume the origin of the coordinate system to be the target nucleus and the $z$-axis to be along the incident momentum. The $x$-axis is in the plane defined by the incident momentum and the polarization vector of the laser field. The angle between the polarization vector and the incident momentum is denoted by $\Theta$. The scattering angle of the fast (scattered) electron and the emission angle of the slow (ejected) electron are denoted respectively by $\theta_{f}$ and $\theta_{e}$. The former is measured in anticlockwise direction, and the latter clockwise. The angle between the scattering plane and the $z x$-plane is referred to by $\phi$ (the azimuth angle).

Figure 1 shows the distribution of the multiphoton TDCS as a function of $l$ (the number of exchange photons) for the two cases where the laser polarization is either parallel or perpendicular to the incident momentum. The target is atomic hydrogen and the emission angles are $\theta_{f}=4^{\circ}, \theta_{e}=45^{\circ}$ and $\phi=0^{\circ}$. The spectra are approximately symmetric about $l=0$. The photon absorption processes $(l<0)$ dominate those of photon emission $(l>0)$; meaning that the system absorbs net energy from the radiation background. The cross sections for both geometries are strikingly different: the TDCS for $\mathcal{E}_{0} \| \mathbf{k}_{i}$ peaks sharply near $l=0$, in agreement with previous studies (cf [13] and references therein). On the other hand, for $\mathcal{E}_{0} \perp \mathbf{k}_{i}$ the cross section is determined by multiphoton processes involving a large number of photons. In both the cases the cut-off (in $l$ ) is determined by the Bessel function $J_{l}\left(\mathbf{D} \cdot \boldsymbol{\alpha}_{0}\right)$ in equation (9): when the argument $\mathbf{D} \cdot \boldsymbol{\alpha}_{0}$ and the order $l$ are approximately equal the value of the function $J_{l}\left(\mathbf{D} \cdot \boldsymbol{\alpha}_{0}\right)$ diminishes rapidly. The physics behind this mathematical observation is the following: $\mathbf{- D}$ is the recoil ion momentum. In the extreme case where $-\mathbf{D}$ is very small, e.g., when the nucleus is a spectator during the collisions, $\mathbf{D} \cdot \boldsymbol{\alpha}_{0}$ is as well very small and effectively the laser field plays a minor role. This is because the energy absorbed by the electrons from the radiation field needs to be converted into a linear momentum via a re-scattering from the massive residual ion. Small $D$ means that such re-scattering processes did not take place and hence the weak influence of the laser field on the outcome of the collision 

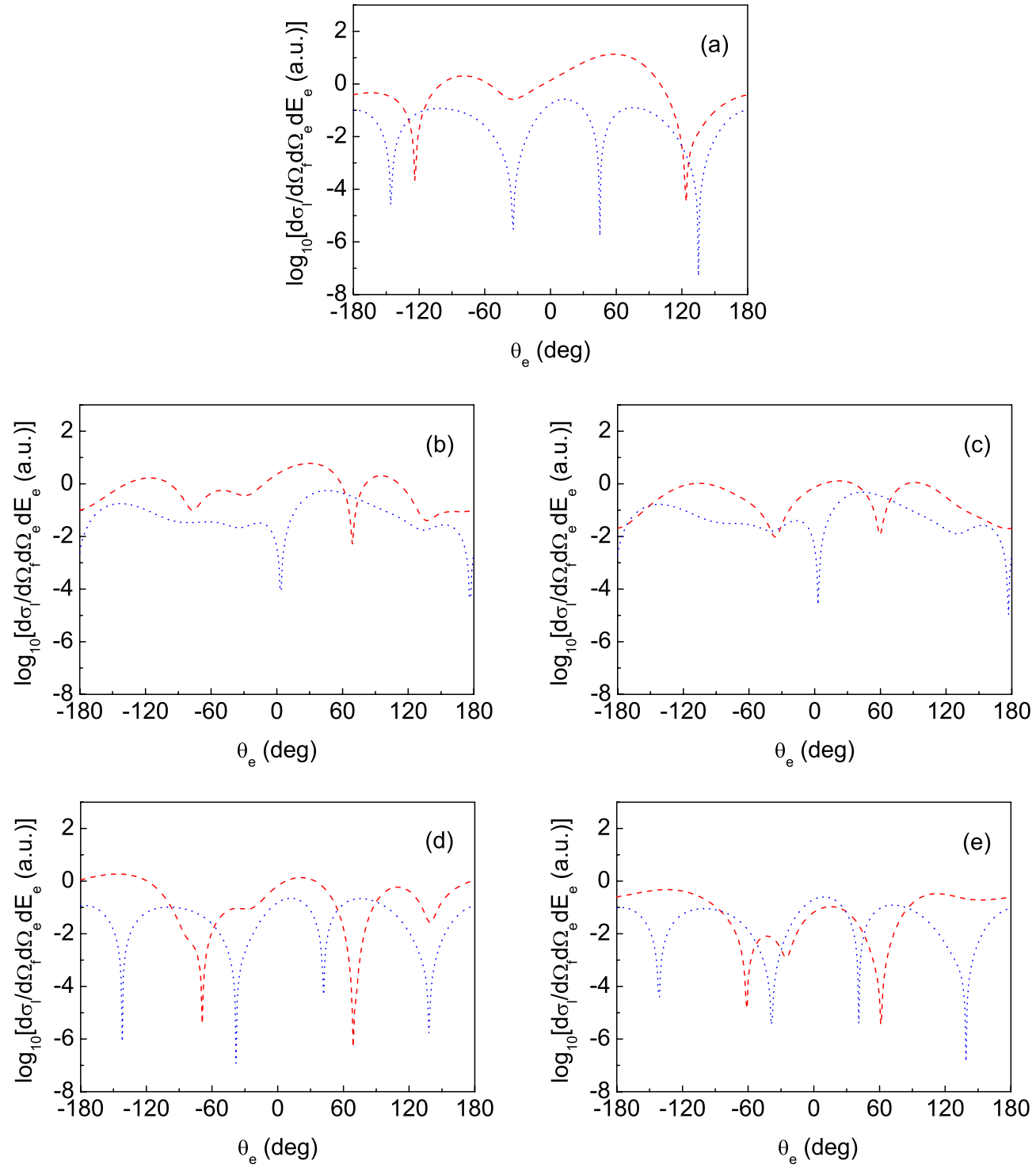

Figure 2. TDCS involving $l$ photon processes. The incident electron is scattered through $\theta_{f}=4^{\circ}$ in the plane spanned by the laser polarization vector and the incident electron momentum vector. (a) $l=0$, (b) $l=-1$, (c) $l=1$, (d) $l=-2$, (e) $l=2$. The kinetic energies of the incident and the ejected electrons and the laser parameters are the same as in figure 1. Dashed line: the TDCS for $\mathcal{E}_{0} \| \mathbf{k}_{i} ;$ dotted line: the TDCS for $\mathcal{E}_{0} \perp \mathbf{k}_{i}$.

process. Conversely, if $D$ is large the scattering processes take place near the nucleus and hence the probability for the electrons to experience a radiative transition is generally much higher, except for $\mathbf{D} \perp \boldsymbol{\alpha}_{0}$, where the electric field of the laser is perpendicular to the recoil ion momentum. These arguments apply also for targets with $Z>1$ and hence the same general behaviour of the cross sections is expected for the one-electron ion targets.

Figure 2 shows the TDCS for a few multiphoton processes. The Bessel function occurring in equation (9) causes the oscillating structure in the results: for a fixed laser field strength, 

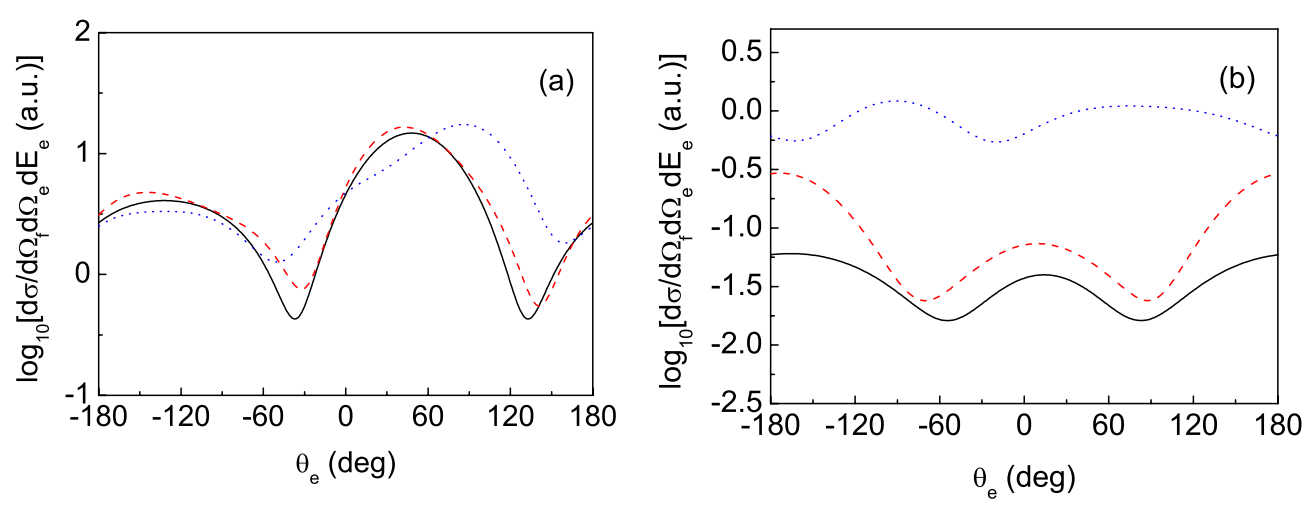

Figure 3. Summed TDCS of $(e, 2 e)$ for hydrogenic ions in Nd:YAG laser field when the fast electron is scattered at $\theta_{f}=4^{\circ}, \phi=0^{\circ}$. The kinetic energy of the incident and ejected electrons are the same as in figure 1. (a) The target is $\mathrm{H}(1 \mathrm{~s})(Z=1)$, the field strength is $\mathcal{E}_{0}=5.0 \times$ $10^{7} \mathrm{~V} \mathrm{~cm}^{-1}$; (b) the target is $\mathrm{He}^{+}(1 \mathrm{~s})(Z=2), \mathcal{E}_{0}=2.0 \times 10^{8} \mathrm{~V} \mathrm{~cm}^{-1}$. Solid line: the TDCS for the laser-free case; dashed line: the TDCS for $\mathcal{E}_{0} \| \mathbf{k}_{i}$; dotted line: the TDCS for $\mathcal{E}_{0} \perp \mathbf{k}_{i}$.

when the magnitude of the total momentum transfer $D$ (the recoil ion momentum) is large the Bessel function argument varies in a wide range when the electron angle is scanned, leading thus to the observed oscillations in TDCS. The curves for $l$ and $-l$ present similar features since $J_{-l}\left(\mathbf{D} \cdot \boldsymbol{\alpha}_{0}\right)=(-1)^{l} J_{l}\left(\mathbf{D} \cdot \boldsymbol{\alpha}_{0}\right)$ [17]. Nevertheless, the cross sections for $l$ and $-l$ are different. The origin of this difference lies in the terms in equation (9) other than $J_{l}\left(\mathbf{D} \cdot \boldsymbol{\alpha}_{0}\right)$.

Figure 3(a) shows the TDCS from atomic hydrogen summed over all multiphoton processes. The hallmarks of the TDCS shape in the laser-free case are the binary peak (with a maximum located around $\theta_{e}=50^{\circ}$ ) and the recoil peak (located at $\theta_{e}=-130^{\circ}$ ) [23]. The binary peak is the result of a direct electron-electron encounter without a significant involvement of the residual ion (via its initial binding the ejected electron may still recoil from the ion). Therefore, the recoil ion momentum $D$ is generally small and hence only a small number of multiphoton processes take place (since $\mathbf{D} \cdot \boldsymbol{\alpha}_{0}$ is small). Nevertheless, for a different direction of the electric field vector, i.e. different directions of $\boldsymbol{\alpha}_{0}$, the scalar product $\mathbf{D} \cdot \boldsymbol{\alpha}_{0}$ may become sizeable and hence the difference seen in figure 3(a) between $E_{0} \| \mathbf{k}_{i}$ and $E_{0} \perp \mathbf{k}_{i}$. It is generally believed [23] that the recoil peak involves significant interaction with the residual ion. On the other hand, in the vicinity of the recoil ion one may expect the probability for radiative transitions to increase [13] leading thus to pronounced modifications of the recoil peak. To explore this idea we performed calculations for the cross sections from targets with varying $Z{ }^{5}$ The final electron energies and emission angles are the same as in figure 3(a). The outcome of the calculations is the following. If $Z$ is increased without changing the strength of the laser field amplitude we find that the cross sections tend to the laser-free case over the whole angular range (results are not shown here for brevity, (cf, however, figure 6(b))). Generally this behaviour is to be anticipated since the increased residual ion field becomes the determining factor. On the other hand, from a physical point of view one has also to take into consideration the increase in multiphoton processes due to an enhanced scattering from the residual ion when $Z$ increases. Therefore based on our results we conclude that this enhancement in multiphoton processes does not outweigh the influence of the enhanced residual ion field strength

5 In principle, when dealing with electron-impact ionization from a charged target one has to account for the Coulomb distortion of the incoming projectile motion. The effect is less prominent for fast projectiles (which is the case here) and is justified in as much as the use of a plane wave for describing the scattered projectile in the final state. 

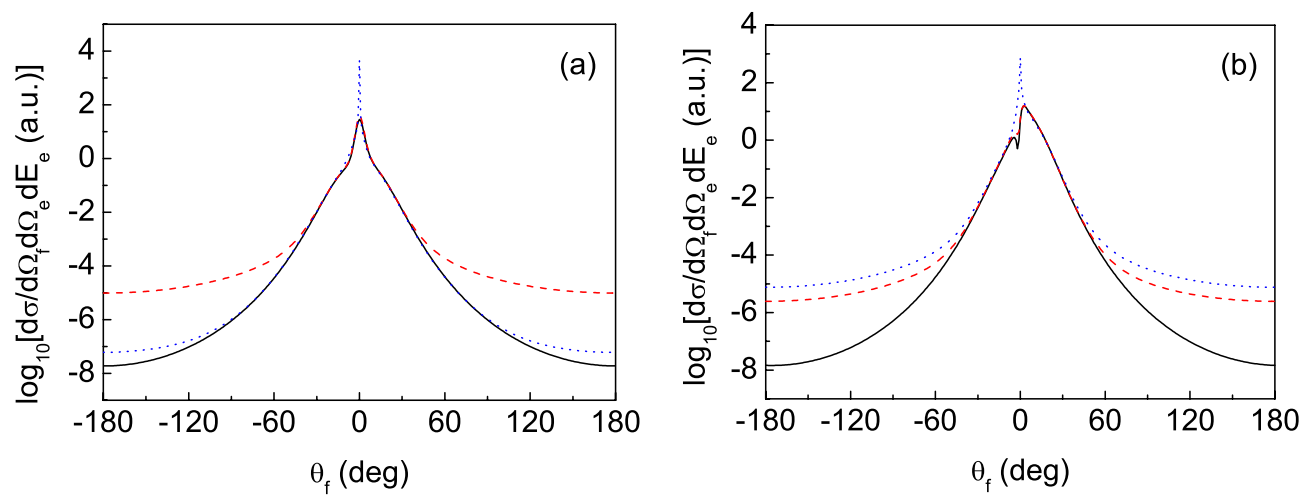

Figure 4. Summed TDCS for atomic hydrogen versus the scattering angle of the fast electron in cases where the initially bound electron is ejected at (a) $\theta_{e}=4^{\circ}$ and (b) $\theta_{e}=60^{\circ}$. The kinetic energies of the incident and the ejected electrons and the laser parameters are the same as in figure 1. Solid line: the TDCS for the laser-free case; dashed line: the TDCS for $\mathcal{E}_{0} \| \mathbf{k}_{i}$; dotted line: the TDCS for $\mathcal{E}_{0} \perp \mathbf{k}_{i}$.

(over the laser field) when $Z$ is increased. This situation changes when the strength of the residual ion field and the laser field strength as felt by the bound $1 \mathrm{~s}$ electron are increased by the same amount (figure 3(b)): figure 3(b) shows the TDCS from the 1s ground state of an ionic target with a charge $Z=2$. The laser field strength is then increased (with respect to figure 3(a)) by a factor of $Z^{2}=4{ }^{6}$ The cross section modification in the perpendicular geometry is far more severe than in the parallel geometry and consists in a substantial ionization enhancement (as compared to the laser-free case). Also the shape of the cross section in the perpendicular geometry is qualitatively different, it resembles roughly previous finding [13], where it has been shown that applying a laser field may result in a splitting in two parts of the binary and the recoil peaks. In our case this splitting is accompanied also by a shift of the positions of the peaks and a changed ratio of the peak maximal values. We remark that figure 3(b) shows the TDCS summed over all multiphoton processes. The $l$ resolved TDCS possess richer structure which is smeared out when performing the sum over $l$.

Figure 4 shows the summed TDCS for atomic hydrogen versus the scattering angle of the fast electron when the slow electron is ejected at $\theta_{e}=4^{\circ}$ and $60^{\circ}$. Since the projectile is fast, it is mainly scattered in the forward direction. The theoretical results provide evidence that when the laser polarization is perpendicular to the incident momentum, the summed TDCS is enhanced considerably in the vicinity of $0^{\circ}$. The reason for this is the following: upon a 'clean' electron-electron (classical) encounter, i.e. if the ion is a spectator, the two electrons emerge perpendicular to each other (due to their equal masses). This implies that for $\theta_{f} \approx 0^{\circ}$ and $\theta_{e}=4^{\circ}$ a scattering of the ejected electron from the residual ion is inevitable. The laser field modifies then strongly the scattering cross sections around $\theta_{f} \approx 0^{\circ}$ because the ejected electron has a small energy and it approaches closely the nucleus where energy from the radiation background is efficiently converted into linear electron momentum.

Figure 5 shows the dependence of summed TDCS on the the azimuthal angle $\phi$ in the geometry $\mathcal{E}_{0} \perp \mathbf{k}_{i}$. We find that when the scattering angle of the fast electron is small, the azimuthal angle dependence is weak; this is because the axial symmetry is only slightly

6 The $Z^{2}$ increase in the field strengths felt by the 1 s electron is due to the enhancement (by $Z$ ) of the charge of the ion and to the decreased mean distance (by $1 / Z$ ) of the bound electron from the nucleus. 

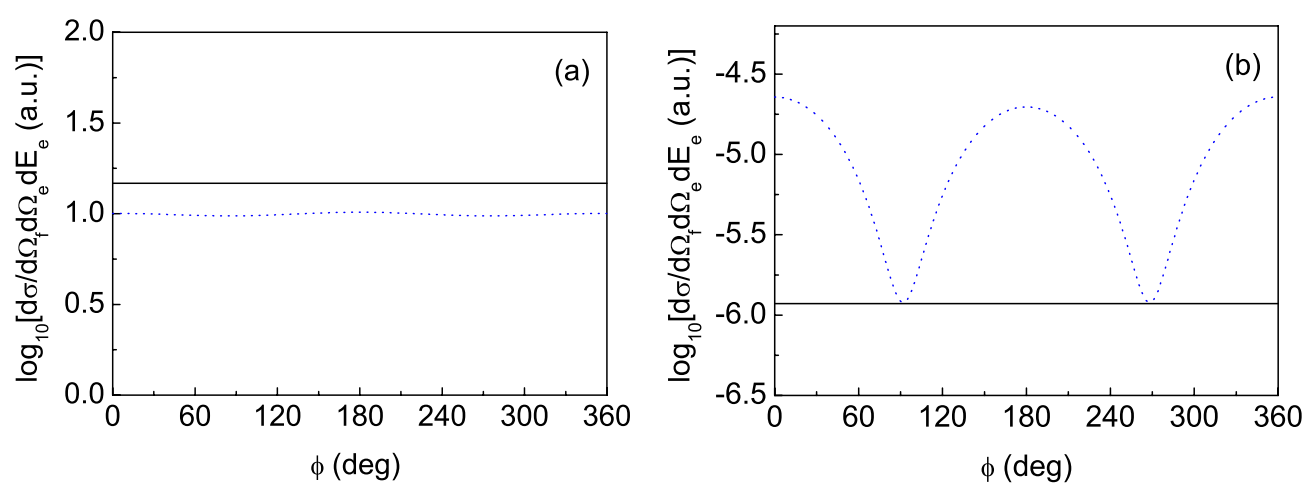

Figure 5. Azimuthal angular dependence of the summed TDCS in atomic hydrogen for $\mathcal{E}_{0} \perp \mathbf{k}_{i}$ at $\theta_{e}=45^{\circ}$ for (a) $\theta_{f}=4^{\circ}$, (b) $\theta_{f}=90^{\circ}$. The kinetic energies of the impinging and the ejected electrons and the laser parameters are the same as in figure 1. Solid line: the TDCS in the absence of the laser; dotted line: the laser modified TDCS.
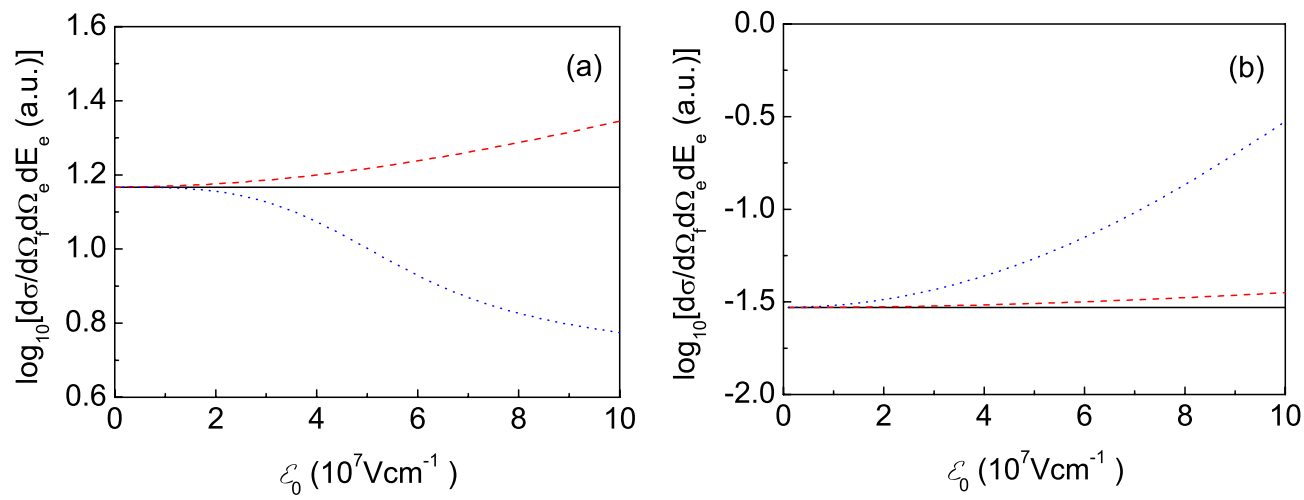

Figure 6. Dependence of the summed TDCS on the field amplitude for the laser frequency $\hbar \omega=1.17 \mathrm{eV}$. The scattering angles of the fast and the slow electrons are respectively $\theta_{f}=4^{\circ}, \theta_{e}=45^{\circ}$. The energies of the incident and the ejected electrons are the same as in figure 1. (a) The target is $\mathrm{H}(1 \mathrm{~s})(Z=1)$, (b) the target is $\mathrm{He}^{+}(1 \mathrm{~s})(Z=2)$. Solid line: the TDCS in the absence of the laser; dashed line: the TDCS for $\mathcal{E}_{0} \| \mathbf{k}_{i}$; dotted line: the TDCS for $\mathcal{E}_{0} \perp \mathbf{k}_{i}$.

broken. However, when the momentum of the fast electron deviates significantly from the $z$-axis, the dependence on the azimuthal angle becomes evident.

Figure 6 shows the dependence of the summed TDCS on the laser field strength. A stronger laser field amplitude and a smaller frequency lead to more pronounced modifications of the TDCS in the presence of the laser. This is because the stronger the laser, the more the states of the free and the bound electrons are distorted (see equations (3)-(6)). This can be inferred from the mathematical formulae by noting that $\mathbf{D} \cdot \boldsymbol{\alpha}_{0}=\left(\mathcal{E}_{0} D / \omega^{2}\right) \hat{\mathbf{D}} \cdot \hat{\mathcal{E}}_{0}$ and that $J_{l}\left(\mathbf{D} \cdot \boldsymbol{\alpha}_{0}\right)$ drops substantially for $l \approx \mathbf{D} \cdot \boldsymbol{\alpha}_{0}$. In figure 6(a) we observe a small influence of the laser field at low field strengths. This is due to the chosen geometry that coincides approximately with the region of the binary peak. As argued above, this situation changes in the recoil peak region where even a weak laser field strength leads to sizeable changes in the cross section. In figure $6(\mathrm{~b})$, we depict the influence of the Coulomb field strength on the cross section for a larger atomic number $(Z=2)$. As $Z$ increases the influence of the laser field on the electrons motion becomes marginal as compared to the action of the Coulomb field of the 


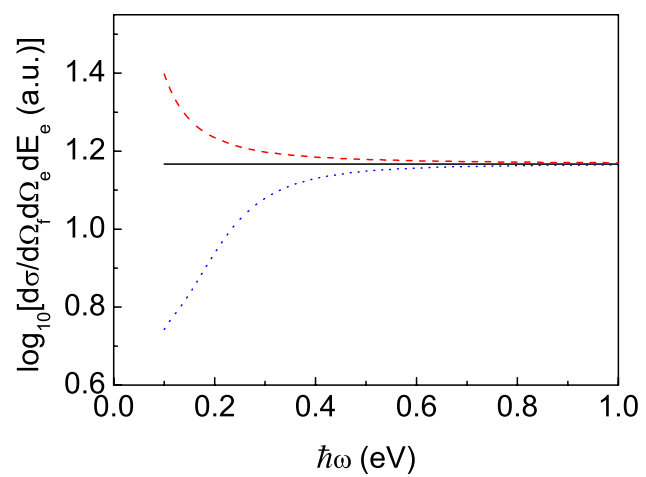

Figure 7. Dependence of the summed TDCS on the field frequency (photon energy) in atomic hydrogen for a fixed field amplitude $\mathcal{E}_{0} \| \mathbf{k}_{i}$. The emission angles and the kinetic energies of both electrons are the same as in figure 5. Solid line: the TDCS for the laser-free case; dashed line: the TDCS for $\mathcal{E}_{0} \| \mathbf{k}_{i}$; dotted line: the TDCS for $\mathcal{E}_{0} \perp \mathbf{k}_{i}$.

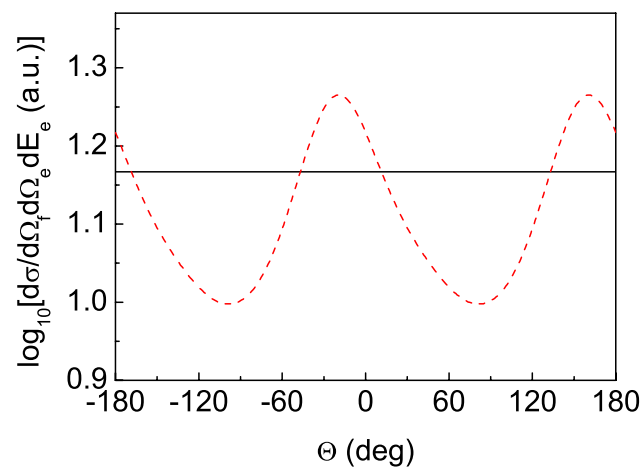

Figure 8. Dependence of the summed TDCS on the laser polarization direction in atomic hydrogen. The laser field strength and the laser frequency as well as the scattering angles and the kinetic energies for both electrons are the same as in figure 1. Solid line: the TDCS in the absence of the laser; dashed line: the laser modified result.

residual ion and hence we observe a decreasing influence of the laser on the cross section with increasing $Z$.

Figure 7 reveals a qualitatively different behaviour of the TDCS as a function of the field frequency and of the field amplitude strength. The trend observed in figure 7 becomes comprehensible by inspecting the dressed states given by equations (3)-(6). The higher the frequency, the less the states are modified by laser. For high laser frequencies the electron is unable to respond to the fast field oscillation and hence the influence of the laser on the cross section is marginal.

Figure 8 shows the dependence of the cross section on the direction of the electric field of the laser. The periodic character is due to the periodic dependence of the argument D. $\boldsymbol{\alpha}_{0}=\left(\mathcal{E}_{0} D / \omega^{2}\right) \cos \Theta$ of Bessel functions which basically determines the number of contributing multiphoton processes.

Figure 9 shows the summed TDCS for atomic hydrogen versus the impact energy. Since the angle and the energy of the slow ejected electron are fixed only the energy of the fast incoming and scattered electron are changed by varying the impact energy. As is clear from figure 9, large laser-induced modifications to the cross section are observed at lower impact 


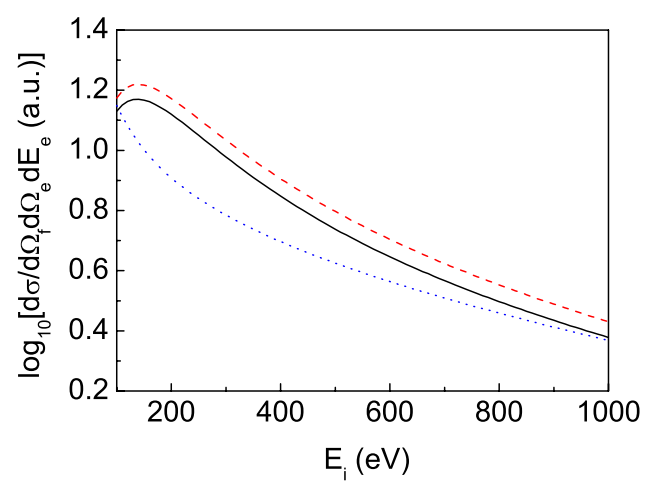

Figure 9. Summed TDCS versus the incident electron energy. The kinetic energy of the slow electron is $E_{e}=3 \mathrm{eV}$. The emission angles of both electrons and the laser parameters are the same as in figure 1. Solid line: the TDCS in the absence of the laser; dashed line: the TDCS for $\mathcal{E}_{0} \| \mathbf{k}_{i}$; dotted line: the TDCS for $\mathcal{E}_{0} \perp \mathbf{k}_{i}$.
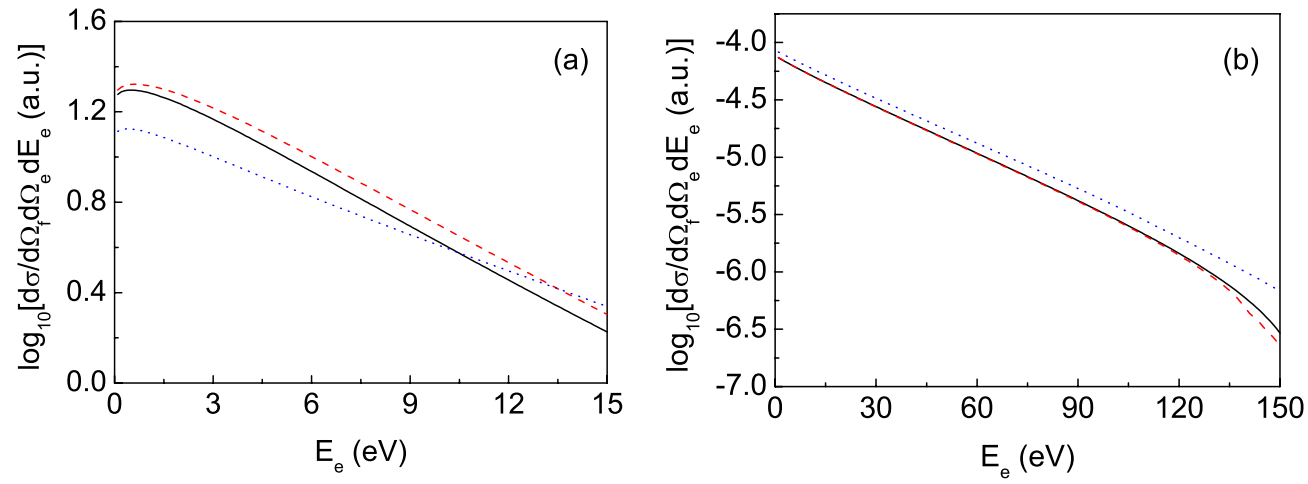

Figure 10. Summed TDCS versus the energy of the slow electron. In (a) we consider atomic hydrogen $\mathrm{H}(1 \mathrm{~s})$ ionized by $150 \mathrm{eV}$ electrons, in (b) the target is $\mathrm{B}^{4+}$, i.e. $Z=5$ and the incident energy is $E_{i}=500 \mathrm{eV}$. The emission angles of both electrons in the final state and the laser parameters are the same as in figure 1. Solid line: the TDCS for the laser-free case; dashed line: the TDCS for $\mathcal{E}_{0} \| \mathbf{k}_{i}$; dotted line: the TDCS for $\mathcal{E}_{0} \perp \mathbf{k}_{i}$.

energies where the laser acts more effectively on the impinging electron. If the incident electron energy is increased the influence of the laser on the projectile electron becomes less important, as readily deduced from the structure of the Volkov states when $E_{i}$ and $E_{f}$ are large. We note that the modifications, observed in figure 9, depend on the properties of the projectile. Within the FBA the projectile is not exposed to the Coulomb field of the residual ion and hence, within the present model, similar dependences on the incident electron energies are expected for hydrogenic targets, regardless of the charge state of the residual ion.

Figure 10(a) displays the summed TDCS for atomic hydrogen versus the slow electron energy. For a parallel orientation of the laser polarization (to the incident electron momentum), the laser effects are more pronounced when the ejected electron energy is increased. This is due to an increased $D$ which opens the possibility for an enhanced multiphoton process, as discussed above. That the laser background can as well suppress the (e, 2e) cross section is demonstrated in figure 10(a) for the perpendicular geometry. This suppression effect is due to the laser-induced modification of the initial-state momentum distribution. On the other hand, 
in the binary region the cross section is strongly dependent on the momentum profile of the initial state and hence on how the laser field affects this profile. Figure 10(b) shows the same situation as in figure 10 (a), however the target is $\mathrm{B}^{4+}$, i.e. $Z=5$. Contrasting figures 10 (a) and (b) we observe that with an increased nuclear charge $Z$ the Coulomb field effects dominate the low-energy electron emission process. However, for high energies of the ejected electrons the $(\mathrm{e}, 2 \mathrm{e})$ process involves inevitably high momentum transfer processes, i.e. close collisions (with respect to the nucleus position). Therefore, we observe a gradually increasing influence of the radiation field on the cross section with increasing electron energies.

\section{Conclusions and final remarks}

Unlike the case of laser-assisted heavy projectile-impact ionization in which the state of the projectile is not modified significantly by the presence of the laser [24], for the electron impact laser-assisted $(e, 2 e)$ reaction, the fast electron exchanges a large number of photons with the radiation field. At large scattering angles, the laser modification of the fast electron state is reflected in considerable changes in the cross sections. A number of effects occurring when the laser is applied can be attributed to the Bessel function that appears in the determining relation (9) for the multiphoton (e, 2e) amplitude. For the laser-free case the initial and final-state wavefunctions of the target electron are orthogonal which, within the FBA, inhibits direct scattering of the projectile from the nucleus. When the laser is switched on, the fielddependent term in the parentheses in equation (6) invalidates this orthogonality argument and consequently the projectile direct scattering from the nucleus (the $-Z / r_{0}$ term) contributes to the $S$-matrix of equation (8).

The numerical results indicate that in the laser-assisted (e, 2e) collision, the photon absorption processes (inverse bremsstrahlung) dominate the photon emission (bremsstrahlung). The angle between the polarization vector of the field and the total momentum transfer of both electrons affects significantly the characteristics of scattering cross sections. When the laser polarization is parallel to the incident direction, the TDCS summed over the multiphoton processes is generally enhanced. When the polarization vector is perpendicular to the incident momentum, the laser modification to the summed TDCS is qualitatively different. At small scattering angles of the fast electron, the laser modified TDCS shows a weak azimuthal-angle dependence. We also explored the dependence of the TDCS on the laser field parameters and on the strength of the residual ion field (by varying $Z$ ). We find that with increasing the strength of the laser fields and lowering the frequencies, the TDCS is modified more effectively. When the laser parameters are fixed and $Z$ is increased the effect of the laser on the TDCS diminishes rapidly.

\section{Acknowledgments}

This work is supported by the National Natural Science Foundation of China under grantnumbers 10475070 and 10074060 . SML thanks the DAAD for the financial support during his stay in Germany.

\section{References}

[1] Mittleman M H 1993 Introduction to the Theory of Laser-Atom Interactions (New York: Plenum)

[2] Francken P and Joachain C J 1990 J. Opt. Soc. Am. B 7554

[3] Ehlotzky F, Jaroń A and Kamiński J Z 1998 Phys. Rep. 29763

[4] Ehlotzky F 2001 Phys. Rep. 345175 
[5] Haug H and Jauho A P 1996 Quantum Kinetics in Transport and Optics of Semiconductors (Berlin: Springer)

[6] Höhr C, Dorn A, Najjari B, Fischer D, Schröter C D and Ullrich J 2005 Phys. Rev. Lett. at press

[7] Ullrich J et al 2003 Rep. Prog. Phys. 661463

[8] Bray I 2000 J. Phys. B: At. Mol. Opt. Phys. 33581

[9] Cavaliere P, Ferrante G and Leone C 1980 J. Phys. B: At. Mol. Phys. 134495

[10] Joachain C J, Francken P, Maquet A, Martin P and Veniard V 1988 Phys. Rev. Lett. 11165

[11] Martin P, Veniard V, Maquet A, Francken P and Joachain C J 1989 Phys. Rev. A 196178

[12] Khalil D, Maquet A, Taïb R, Joachain C J and Makhoute A 1997 Phys. Rev. A 564918

[13] Makhoute A, Khalil D, Maquet A and Taïb R 1999 J. Phys. B: At. Mol. Opt. Phys. 323255

[14] Volkov D M 1935 Z. Phys. 94250

[15] Berestetskii V B, Lifshitz E M and Pitaevskii L P 1982 Quantum Electrodynamics (Course of Theoretical Physics vol 4) 2nd edn (Oxford: Reed)

[16] Kornev A S and Zon B A 2002 J. Phys. B: At. Mol. Opt. Phys. 352451

[17] Abramowitz M and Stegun I A 1972 Handbook of Mathematical Functions with Formulas, Graphs, and Mathematical Tables (Whashington, DC: United States Department of Commerce)

[18] Li S-M, Chen J and Zhou Z-F 2002 Eur. Phys. J. D 19157

[19] Gradshteyn I S and Ryzhik I M 1994 Tables of Integrals, Series, and Products (New York: Academic)

[20] Akhiezer A I and Merenkov N P 1996 J. Phys. B: At. Mol. Opt. Phys. 292135

[21] Akhiezer A I and Berestetsky V B 1969 Quantum Electrodynamics (Moscow: Nauka)

[22] Berakdar J 1997 Phys. Rev. A 56370

[23] Ehrhardt H, Jung K, Knoth G and Schlemmer P 1986 Z. Phys. D 13

[24] Li S-M, Chen J and Zhou Z-F 2002 J. Phys. B: At. Mol. Opt. Phys. 35557 